\title{
Identifying Bubble Occurrence during Pool Boiling Employing Acoustic Emission Technique
}

\author{
Taihiret Alhashan ${ }^{1}$, Abdulmajid Addali ${ }^{2}$, Joao Amaral Teixeira ${ }^{3}$, Said Elhashan ${ }^{4}$ \\ 1,2,3 School of Energy, Cranfield University, Cranfield, Beds. MK43 OAL. \\ ${ }^{4}$ Higher Institute for Mechanical Engineering, Tripoli-Libya
}

Email: t.a.alhashan@cranfield.ac.uk

\begin{abstract}
This paper reports the results of a study for the early detection of bubble formation during the boiling process using acoustic emission. The feasibility of using $A E$ technology to detect and monitor early bubble formation during pool boiling is assessed, and the results show that $A E$ technology is an affective tool for this purpose. There is a clear correlation between the AE signal levels and height of the water level above the heated surface during the boiling process. The different types of heated fluid influence $A E$ energy levels during the bubble formation process. Statistically, it was found that the best $A E$ parameters to indicate bubble formation were AE-RMS, AE-Energy and AE-Amplitude.
\end{abstract}

Keywords: pool boiling, bubble formation, acoustic emission

\section{Introduction}

Cavitation is an undesirable phenomenon generated by pressure waves associated with bubble formation and collapse in a liquid and can cause serious damage to hydraulic components, increasing maintenance costs, decreasing the life of equipment, and reducing production and revenue. Cavitation occurs when a liquid is subjected to pressure fluctuations which cause pockets of the fluid to experience local pressures which are equal to or lower than the saturated vapour pressure at the given temperature [1][2]. Vapour bubbles form and collapse into micro-jets leading to erosion and pitting of material surfaces, and causing high levels of noise and vibration, indeed the pressure pulse emitted by the bubble collapse creates shock waves [3][4]. The operation of a centrifugal pump under bubble cavitation conditions for a significant time causes pitting and erosion of the impeller vane [2]. When the bubble cavities collapsed, they produced sound and dissipated energy in the water $[5]$. 
Cavitation collapse depends on the number and size of the bubbles. In other words, large numbers of small bubbles create a high-frequency noise and vibration, while a limited number of large bubbles produce a low-frequency noise and vibration [6]. A bubble in order of $6 \mu \mathrm{m}$ diameter will produce sound with a peak frequency of about $500 \mathrm{kHz}$, and $10 \mu \mathrm{m}$ diameter yields $300 \mathrm{kHz}$, as shown in Figure 1, which suggest the peak frequency of the pulse is inversely proportional to the bubble diameter [7].

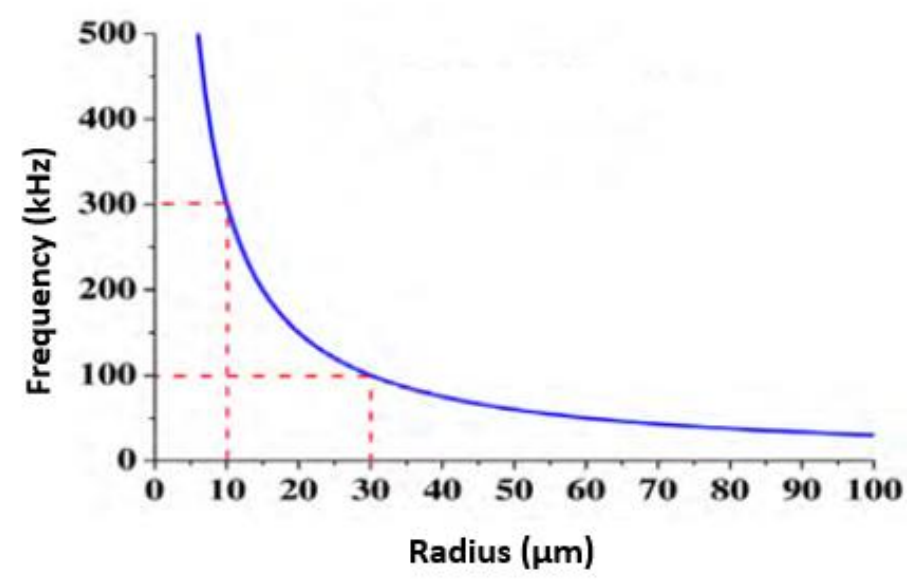

Figure 1: Relation between frequency and bubble size [7].

To decrease the damage caused by cavitation, bubble formation must be monitored and diagnosed in rotating machines and valves [2].

Alhashan et al., [8] used the $A E$ technique to monitor bubble formation during the boiling process. They found a clear association between increasing $A E$ levels and bubble formation. Benes and Uher [9] found that the parameters of the AE signal correlated with overheating during heat transfer and it was established that the $\mathrm{AE}$ signal could be used to predict the boiling phenomena. Carmi et al., [7] used AE in a flow boiling experiment for detection of bubble transit, noting the possibility of using $A E$ for the detection of bubble dynamic events at an early stage in the boiling process. Baek et al. [10] found that bubble density increases with the increase of the liquid temperature. Furthermore, they identified a relation between the water boiling phenomenon and the $\mathrm{AE}$ signals in a transparent glass cell at 1 bar. The $\mathrm{AE}$ technique has been used to diagnose bubble formation and monitor bubble departure from the heating surface of a boiler to the top of the water free surface. It was established that the AE-RMS is a sensitive, reliable and robust parameter for monitoring bubble dynamics its propagation to the water surface during the boiling 
process [11]. This work will focus on monitoring of bubble activity and their departure from heating surface to the water free surface, where finally the bubbles burst at free surface.

In an investigation of two-phase flows, Masjedian et al., [1] used two methods, characteristic diagrams and acoustic analysis, in the monitoring and diagnosis of cavitation phenomena in globe valves. They found good agreement between the two methods at acceptable levels of accuracy. Jaubert et al., [12] noted that $A E$ is a good method for the detection of cavitation phenomena in pumps and valves. This detection can be at an early stage, which makes it possible to study incipient cavitation phenomena. Alfayez et al., [13] demonstrated the use of AE technique for the detection of cavitation in centrifugal pumps and found that this was a method to determine the best efficiency point. Neill et al., [4] used AE technology for the monitoring and detection of cavitation phenomena in a centrifugal pump and claimed it was a more accurate method than using vibration measurement. Osterman et al., [14] used a visualisation method for the detection of incipient cavitation and made a comparison with pressure oscillations measured by a hydrophone on various openings of a valve. They found the visualisation method was more accurate than hydrophone measurement. Shuib [15] found that when bubble size and liquid viscosity increase, the level of $A E$ signals also increase. One particular item of interest in this programme was the investigation of $A E$ energy radiated from bubble formation. The bubble formation phenomena has potential energy which depends on the pressure within the bubbles and their volume.

Acoustic Emission is a physical phenomenon that occurs where transient high frequency elastic waves are emitted by a sudden release of energy from local sources within the body of a solid or liquid, such as might occur in turbulent flow or with cavitation [16][17][18]. Application of $A E$ is not limited to detection and diagnosis of cavitation in hydraulic systems such as centrifugal pumps and valves; it can be used as a monitoring tool for different types of industries such as petroleum engineering using gas-liquid pumps [19], mechanical processes such as bearings and gearbox faults [20], and chemical processing plants [21].

Here, the $A E$ technique is applied to the monitoring and detection of bubble formation during pool boiling while the bubbles are still in their early stages of 
development [8]. To date, published work shows few attempts to apply the $A E$ technique to the monitoring and detection of bubble formation in pool boiling [9][7]. There are many different ways of monitoring and detecting bubble formation in boiling processes, valves and pumps, including vibration, but $A E$ is a useful technique because its frequency range is about $100 \mathrm{kHz}$ to $1 \mathrm{MHz}$ [22][23] so the range of frequency for the $A E$ sensor is above the limit of human hearing and above most environmentally generated noise.

Most of the published reports on bubble formation and collapse that made use of $A E$ methods were to observe cavitation in centrifugal pumps and valves. Here, this area of research has been expanded to assess the feasibility of the use of the $A E$ technique for monitoring bubble formation in boiling processes. Heat transfer in socalled boilers in used in many industries such as chemical, manufacturing and power plants. The capability for early detection and diagnosis of bubble formation during the boiling process to identify such phenomena as overheating is also relevant to nuclear safety and many another industrial processes [7][24].

Bubble formation is considered one of the most common causes of failure in process systems and the early detection and of bubble formation during fluid transportation in pipes, valves and centrifugal pumps would be immensely beneficial. A condition monitoring programme that could successfully detect the early signs of bubble formation to be used as a warning signal for control purpose to avoid the consequence of bubble collapse (cavitation) is highly desirable. This investigation addresses the applicability of $A E$ techniques to detect bubble formation characteristics (such as bubble size, bubble generation rate, and detachment of bubbles during pool boiling) to monitor and detect liquid properties and the liquid level above the heated surface. The boiling process provides a good opportunity to study bubble formation due to the controlled increase of liquid temperature. In addition, this will provide a good opportunity to characterise and differentiate between bubble formation and its bursting at the free surface during the boiling process. Finally, this research will provide a new reference for the use of the $A E$ technique for monitoring of bubble activities in process systems. 


\section{Mechanisms of Acoustic Emission Created from Bubbles}

Bubble formation, collapse and burst generate pressure waves which can be detected within a wide frequency band. The size of the bubble can be calculated using Equation (1) for the natural frequency of oscillation of the bubble, derived by Minneart [25].

$$
f_{0}=\frac{1}{\pi d} \sqrt{\frac{3 \gamma P_{0}}{\rho}}
$$

Where, $f_{0}$ the resonance frequency of the bubble, $d$ is the bubble diameter, $\gamma$ is the polytropic constant of the gas inside the bubble, $P_{0}$ is the hydrostatic pressure and $\rho$ is the liquid density.

The sound caused by bubble occurrence, oscillation and burst at the free surface is dependent on the bubble's size. The period of the pulse produced by bubble collapse and burst is very short, in the order of ms [13]. For pool boiling The bubble's development can be divided into five stages: 1) bubble formation at the heat exchange surface, 2) initial bubble increase in size, 3) bubble coalescence, 4) splitting of bubbles, and 5) bubble bursts at the free surface [15][16]. Neill et al., [4] found that the energy density created by bubble formation is high and includes associated shock waves in the surrounding liquid which can be observed using an AE sensor [26]. Leighton et al., [27] concluded that many sources of sounds in oceans were caused by pressure waves generated by gas bubbles inside the liquid. It is known that the pressure pulses associated with bubble formation and bursting at the surface of the liquid act as sources of acoustic emission [28][29]. Bubble activity as a source of $A E$ and acoustic energy release into the surrounding fluid from the bubble formation and collapse have been used to determine fluid properties [30]. In pool boiling the highest acoustic emission occurs as the bubbles are detached from the heated surface [31]. Blanchard et al., [32] observed that such bubbles coalesce in tap water more readily than in sea water. Abe [33] found that sea water foams more than fresh water. and that bubbles produced in sea water are smaller and continue for longer than in pure water.

Ceccio et al., [34] confirmed there is a significant difference between bubble occurrence in salt water and fresh water. It was noted that the acoustic emission 
from bubble cavitation in fresh water was lower than that produced by salt water. Additionally, they pointed out that small bubbles provided higher frequency acoustic emission compared to large bubbles. Bubbles of similar size created similar acoustic emission regardless whether they were in salt water or fresh water, and the chemical differences did not appear to influence the sound directly [34].

\section{AE parameters}

Root Mean Square (RMS) is a measure of the continuously varying $A E$ signal "voltage" into the AE system. It is an electrical engineering power term defined as the rectified, time averaged signal, measured on a linear scale and recorded in volts. This measure is often used for signal analysis [18][35]. RMS can be calculated as:

$$
R M S=\sqrt{\frac{X_{1}^{2}+X_{2}^{2}+\ldots \ldots .+X_{N}^{2}}{N}}
$$

Where, $X_{1}, X_{2} \ldots \ldots X_{N}$ are sampled values of the voltage, and $N$ is the total number of samples.

Energy, $\mathrm{E}$, is defined as the integral of the square of the voltage signal $(\alpha)$ over the test duration (T) and calculated using the Equation (3) [18][36].

$$
E=\int_{0}^{T} \alpha^{2}(t) d t
$$

Counts are the number of times the AE signal crosses the detection threshold. Also known as ring down counts or threshold crossing counts [18].

$A$ hit is the detection and measurement of an $A E$ signal and depends very much on the threshold level [18].

AE-Amplitude is the maximum (positive or negative) value of the AE signal during an AE hit [18].

The threshold feature records the value of the threshold at the time of an AE hit.

\section{Experimental Work}

This investigation was built on previous work in this area [7][8]. In these experiments, a cylindrical vessel was used to separate the bubble formation and bursting regions. Three different types of water and three different liquid levels were used during the pool boiling tests, as shown in Table 1. 
Table 1: Base experimental parameters for boiler tests.

\begin{tabular}{|l|c|}
\hline \multicolumn{1}{|c|}{ Water Type } & $\begin{array}{c}\text { Water Level }(\mathbf{m m}) \text { above } \\
\text { heated surface }\end{array}$ \\
\hline Tap Water & $100-200-350$ \\
\hline Demineralized Water & \\
\cline { 1 - 1 } Salt Water (5g/L) Concentration & \\
\hline
\end{tabular}

The boiling tests were performed using a general purpose test-rig, as shown in Figure 2. It consisted of a stainless steel water boiler of $270 \mathrm{~mm}$ inner diameter and $440 \mathrm{~mm}$ in height. The maximum capacity of the boiler is 26 litre. It is integral with a heater, located at the bottom, and used to heat the water inside the boiler. The circular heater has an external diameter of $150 \mathrm{~mm}$. Constant electrical power of $2500 \mathrm{~W}$ was supplied to the boiler heater throughout the experiments.

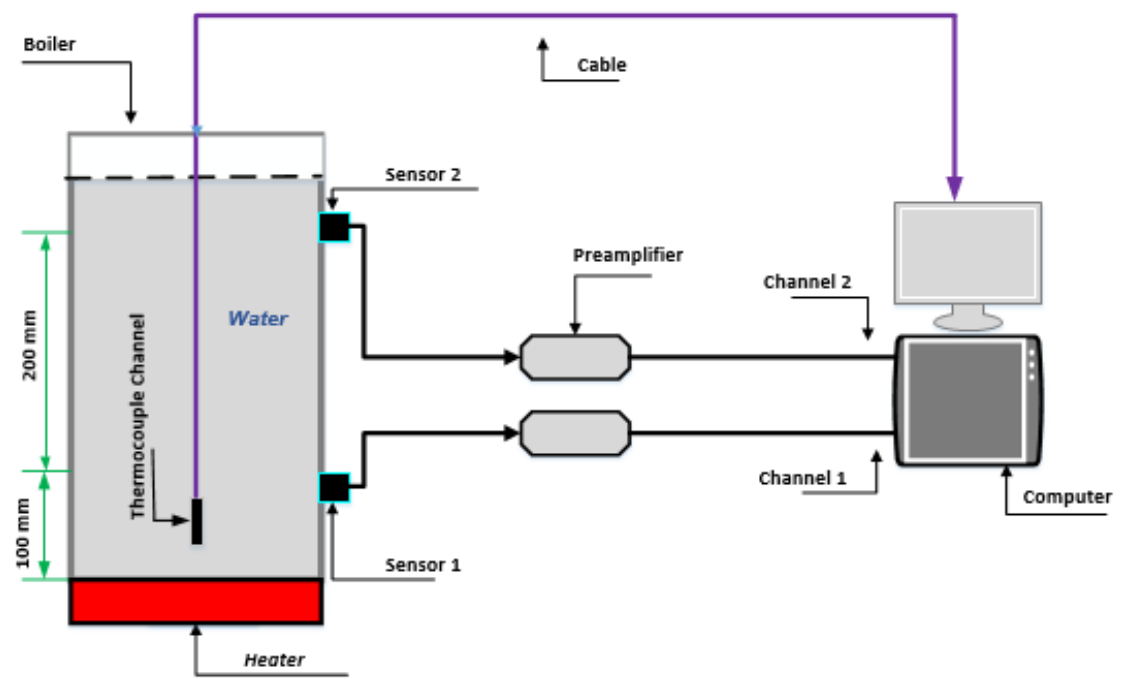

Figure 2: Schematic of experimental arrangement for pool boiling.

Two commercially available piezoelectric AE sensors (Physical Acoustic Corporation type "PICO") with an operating range of $100-1000 \mathrm{kHz}$ were used. The sensors were attached to the external surface of the boiler using superglue, as shown in Figure 2. The AE sensors were $200 \mathrm{~mm}$ vertically apart. The first channel was attached 100 $\mathrm{mm}$ above the bottom surface, to detect the initiation of bubble formation. Channel 2 was positioned $200 \mathrm{~mm}$ above channel 1 to monitor bubble burst and oscillations 
when the bubbles are at the water surface. Measurements of the water temperature were made continuously every second using a thermocouple that was fixed inside the pool boiling $5 \mathrm{~mm}$ above the surface of the heater, see Figure 2. The acoustic sensors were linked to a data acquisition system by a preamplifier, set at $40 \mathrm{~dB}$ gain. The system was set to continuously acquire $A E$ waveforms at a sampling rate of 2 $\mathrm{MHz}$. The software (signal processing package "AEWIN") is incorporated within a PC to monitor $\mathrm{AE}$ parameters such as $\mathrm{AE}-\mathrm{RMS}$ and $\mathrm{AE}$-Energy (recorded at a time constant of $10 \mathrm{~ms}$ and a sampling rate of $100 \mathrm{~Hz}$ ).

The author chose a threshold level of $36 \mathrm{~dB}$, after observing that the $\mathrm{AE}$ sensors still recorded some background noise when the level was set between 28 and $32 \mathrm{~dB}$. $A E-h i t s$ measuring just background noise at a range of five threshold levels are plotted in Figure 3. At $34 \mathrm{~dB}$, zero noise was recorded, but the threshold value was set at $36 \mathrm{~dB}$ to ensure consistent results throughout the test period.

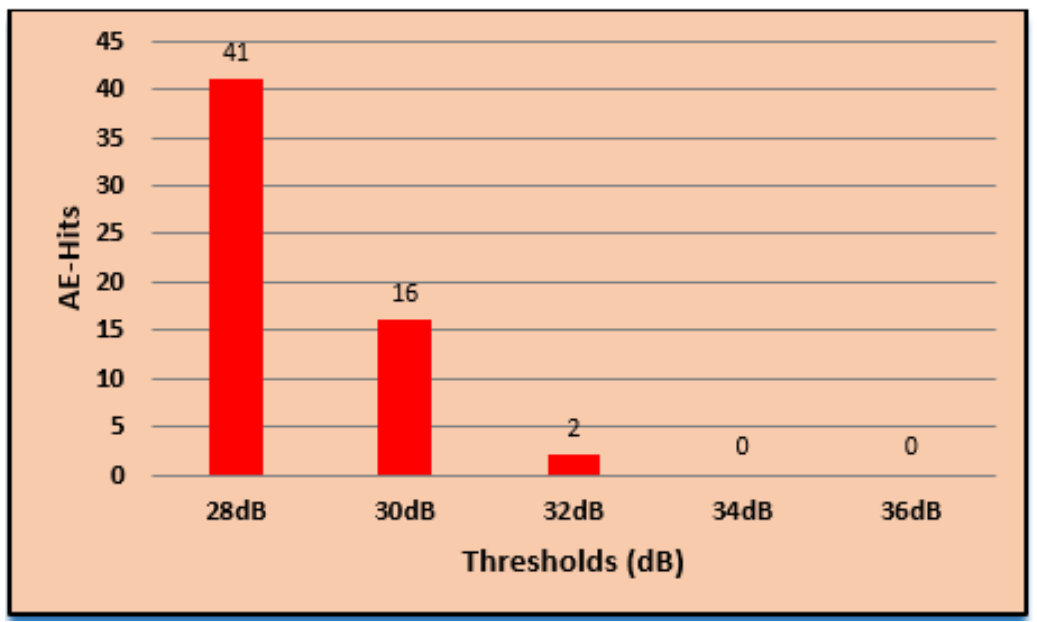

Figure 3: Threshold levels test for operational background noise.

\section{Results Observations and Discussion}

\subsection{Calibration test}

The calibration test was undertaken to quantify the attenuation properties of the boiler vessel material. Attenuation is any reduction (or loss) in the AE levels strength, and it is expressed in decibels (dB's) [23][37][38]. Attenuation tests were carried out before the laboratory experiments. Hsu-Nielsen sources were used for the attenuation tests. These tests consist of breaking a $0.5 \mathrm{~mm}$ diameter pencil lead about $3 \mathrm{~mm}(+/-0.5 \mathrm{~mm})$ from its tip by pressing it against the outside surface of the container. With the discovery threshold was set at $36 \mathrm{~dB}$ the lead break test was 
performed at heights of $50 \mathrm{~mm}, 100 \mathrm{~mm}, 150 \mathrm{~mm}$ and $200 \mathrm{~mm}$ above sensor 1 . furthermore,break test was performed at the same heights below sensor 2 . Then an average value of the maximum signal amplitude of five pencil breaks from each location was calculated, as shown in Figure 4. Signal amplitude and relative attenuation have been computed using the following equation (4) [18][38]:

$$
\text { Attenuatio } n(d B)=20 * \log _{10}\left(\frac{V_{s}}{V_{d}}\right)
$$

Where $V_{s}$ and $V_{d}$ are the signal voltages at the signal source location and at the signal destination location respectively. Measurements revealed that the AE signals generated on the surface of the boiler are attenuated with increasing distance from the source as expected and shown in Figure 4.

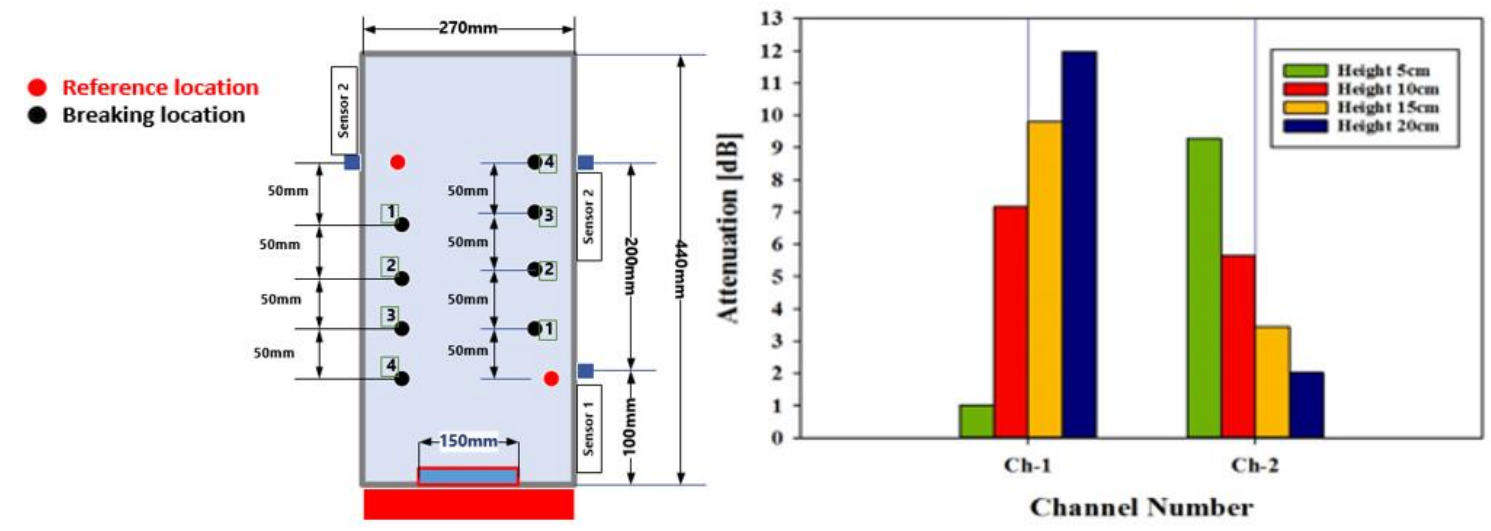

Figure 4: Relative attenuation at four different heights.

\subsection{Monitoring of bubble formation using tap water}

In this investigation, tests were undertaken for three levels of water, at $100 \mathrm{~mm}$ (level with sensor 1), $200 \mathrm{~mm}$ (a level midway between sensor 1 and sensor 2), and 350 $\mathrm{mm}$ (a level $50 \mathrm{~mm}$ above sensor 2). For this particular study, one experimental case is presented, with tests undertaken three times for each of the three levels. Tests were terminated once the water temperature reached the boiling point of $100^{\circ} \mathrm{C}$. The onset water temperature condition for all tests was recorded as being a temperature $\left(20^{\circ} \mathrm{C}\right)$. Continuous observations were made of $\mathrm{AE}-\mathrm{RMS}(\mathrm{mV})$ and temperature $\left({ }^{\circ} \mathrm{C}\right)$ with time (sec), and the different trends identified. Results for the boiler filled with water up to a depth of $200 \mathrm{~mm}$, are presented in Figure 5. Note, the water surface level was $100 \mathrm{~mm}$ below sensor 2 . 


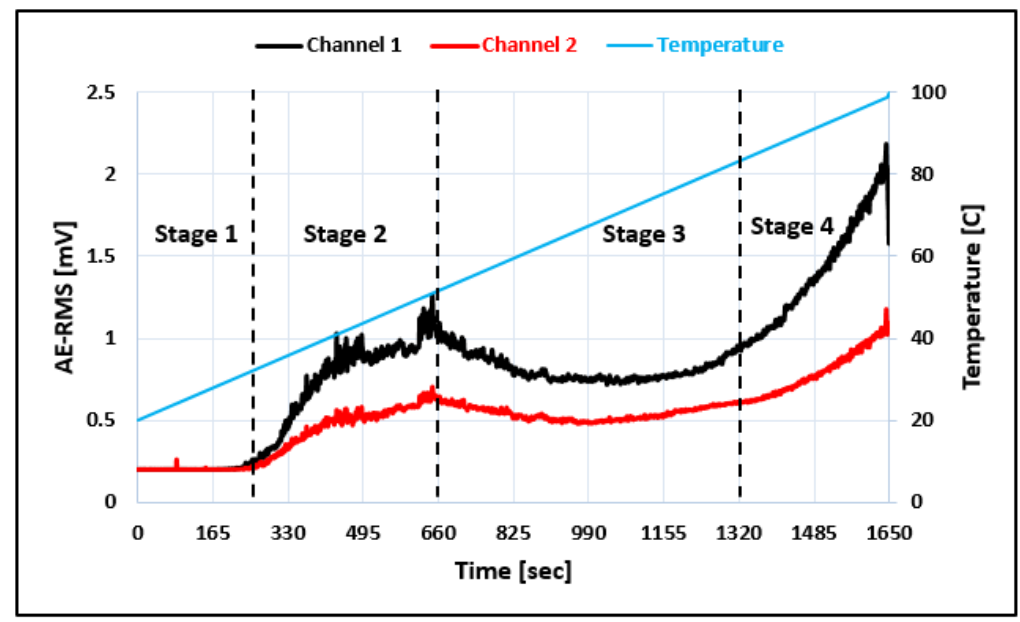

Figure 5: Measured temperature, and AR-RMS signals for boiling test with tap water (water level $200 \mathrm{~mm}$ ).

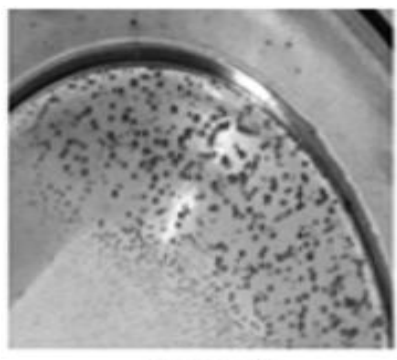

Stage 1

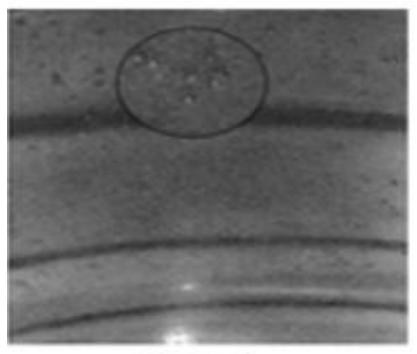

Stage 3

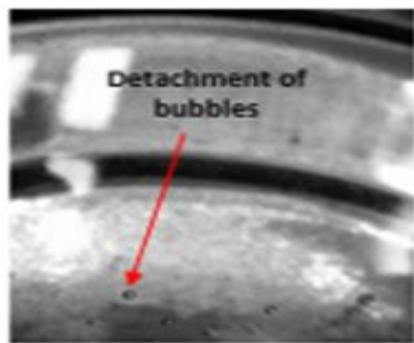

Stage 2

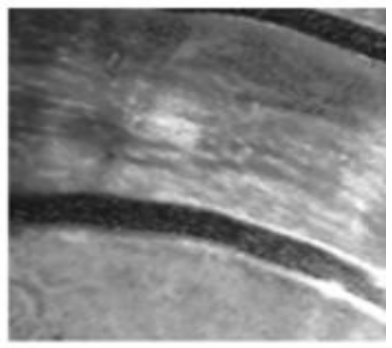

Stage 4

Figure 6: Bubble mechanisms in different stages zones for water level of $200 \mathrm{~mm}$.

Figure 5 above is divided into four different stages. During the first stage, which is between 0 and around $245 \mathrm{~s}$, the heat transfer mechanism is natural convection and few bubbles to form on the heated surface. The measured water temperature rose from its initial value of $22^{\circ} \mathrm{C}$ to about $35^{\circ} \mathrm{C}$. At the second stage, between 245 and $660 \mathrm{~s}$, isolated vapour bubbles form at about $40^{\circ} \mathrm{C}$ on the heated surface, break away and rise through the water bursting when they reach the surface. In this stage, there is a substantial increase in the value of AE-RMS in both channels. The value of the $\mathrm{AE}$ signal in channel 1 is higher than that of channel 2, $(1.3$ and $0.7 \mathrm{mV}$ respectively), because the position of sensor 2 is $100 \mathrm{~mm}$ above the water surface and so does not receive direct $A E$ transmission through the water. In addition, for tap 
water, gas voids appear on the boundaries between the water and the small particles suspended in the water; a phenomenon called heterogeneous nucleation.

As the bubbles begin to form, and then collide with each other, some combine to produce larger bubbles. Buoyance forces cause the bubbles to rise and as they do so the water pressure surrounding them decreases and they grow so that the buoyancy force increases and they accelerate upwards [31].

During the third stage, between 660 and around $1150 \mathrm{~s}$, the value of measured AERMS for both channels decreases. During this period, the sensible heat changes to latent heat. Not all the bubbles detach from heated surface to the surrounding water, some combine with adjacent bubbles and stick to the heated surface, causing an isolating layer, as shown in Figures 5 and 6 . This layer is responsible for attenuation of AE-signals. At about $1100 \mathrm{~s}$, between 75 and $83^{\circ} \mathrm{C}$, the value of $A E-R M S$ is a minimum in both channels. After this both measured AE-RMS levels begin to increase.

Finally, in stage four, as the water approaches the boiling stage between 83 and $100^{\circ} \mathrm{C}$, there is a gradual but quite steep increase in the value of $A E$ level from 0.8 $\mathrm{mV}$ to approximately $2.2 \mathrm{mV}$ in channel 1 , due to the increase in bubble formation because of heat gained by the water. The number of bubbles reaching the top surface of the water increases, and large bubbles with higher interna; energy levels start to burst on the water surface. Husin et al. [39] found that AE technology is able to detect single bubble burst at the free surface of the liquid. Moreover, they confirmed that $A E$ amplitude and energy of bubble burst at the free surface increases as bubble size increases.

\subsection{Waveform analysis of AE amplitude using tap water}

The $A E$ waveforms measured by sensor 1, when the water level was $200 \mathrm{~mm}$, showed different characteristics depending on the stage. Typical AE waveforms are shown in Figure 7. During stage 1, at $100 \mathrm{~s}$, between 20 and $30^{\circ} \mathrm{C}$, there is virtually no $A E$ signal because only a few bubbles start to form but generally do not detach from the bottom surface of the boiler into surrounding water. During the second stage, it was observed that the value of AE-Amplitude increased to as high as $6 \mathrm{mV}$ at $495 \mathrm{~s}$. At this stage, more bubbles are formed and detach from the heating surface, some collide with others and combine to produce larger bubbles. 

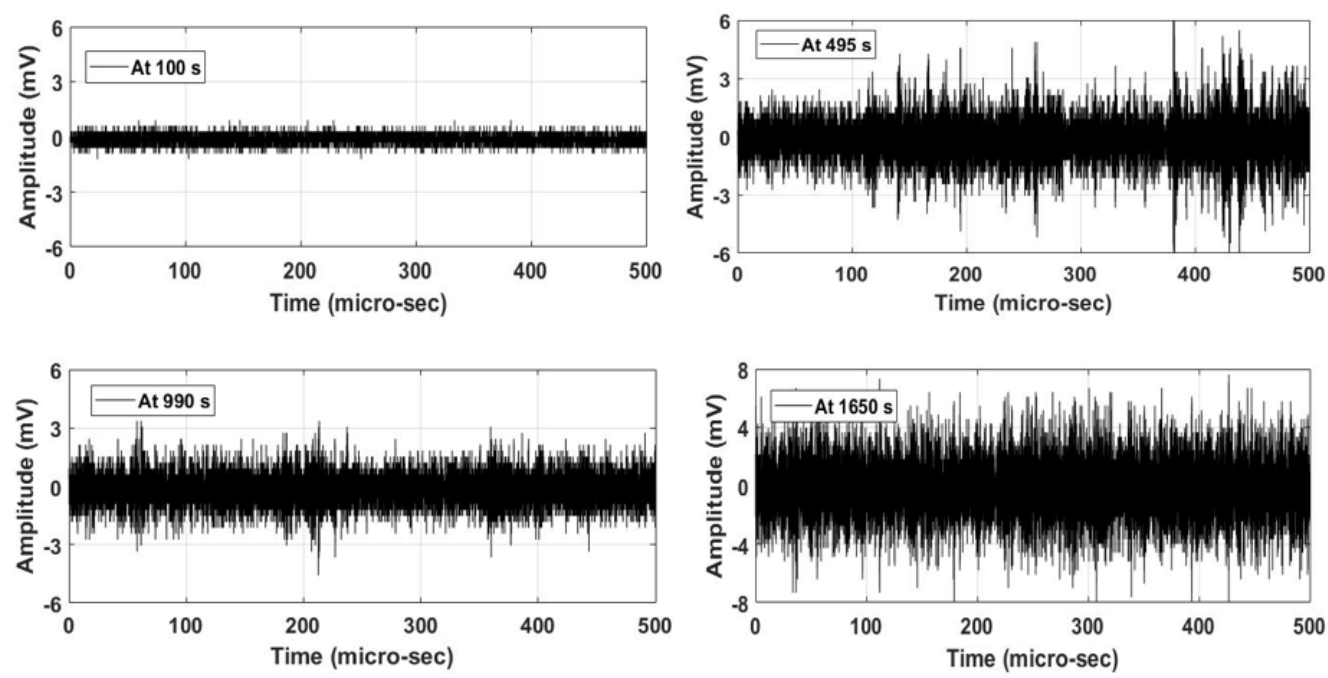

Figure 7: AE waveforms associated with the tap water level of $200 \mathrm{~mm}$ for channel 1.

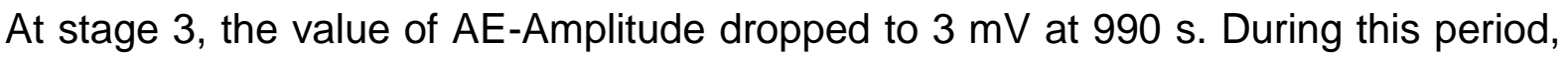
some bubbles combined with adjacent bubbles and adhered to the heated surface causing attenuation of the $A E$ signals. During stage 4, the value of $A E-A m p l i t u d e$ increased until it reached about $8 \mathrm{mV}$; the temperature of the water increased, and large bubbles began to burst on the water surface and this continued until the end of the test. As the size of bubbles increased so did their internal energy.

\subsection{Frequency domain analysis of bubble formation using tap water}

The Fast Fourier Transform (FFT) was used to identify frequency characteristics for bubble activity in tap water with a water level of $200 \mathrm{~mm}$, the results are shown in Figure 8. The measured AE data was processed to estimate the power spectrum of the AE signal arising from bubble activity. The spectrum obtained from the FFT was used to monitor bubble formation during pool boiling. During stage 1 there was a small peak at about $2300 \mathrm{kHz}$, indicating the presence of only a few bubbles forming on the heating surface. These tended to stick to the heated surface where the temperature was still relatively low. During this stage the heat transfers from heated surface to the water was by natural convection.

At stage 2, the value of $A E$ amplitude at the peak frequency of about $2300 \mathrm{kHz}$ increases substantially to approximately $0.2 \mathrm{mV}$. In this stage, the temperature increases, the heat flux increase, and bubbles start to form at a faster rate and to detach from the heated surface. There is a small peak at $1250 \mathrm{kHz}$ which suggests 
the presence of larger bubbles contributing to the AE signal, these might be surface bursts. At stage 3 , the peak value of $A E$ amplitude level decreased gradually to approximately $0.1 \mathrm{mV}$. The peak at $1250 \mathrm{kHz}$ also decreased in magnitude. During this stage, some bubbles combined with others and stuck to heated surface and formed an insulating layer. This layer reduced the levels of the AE signals.
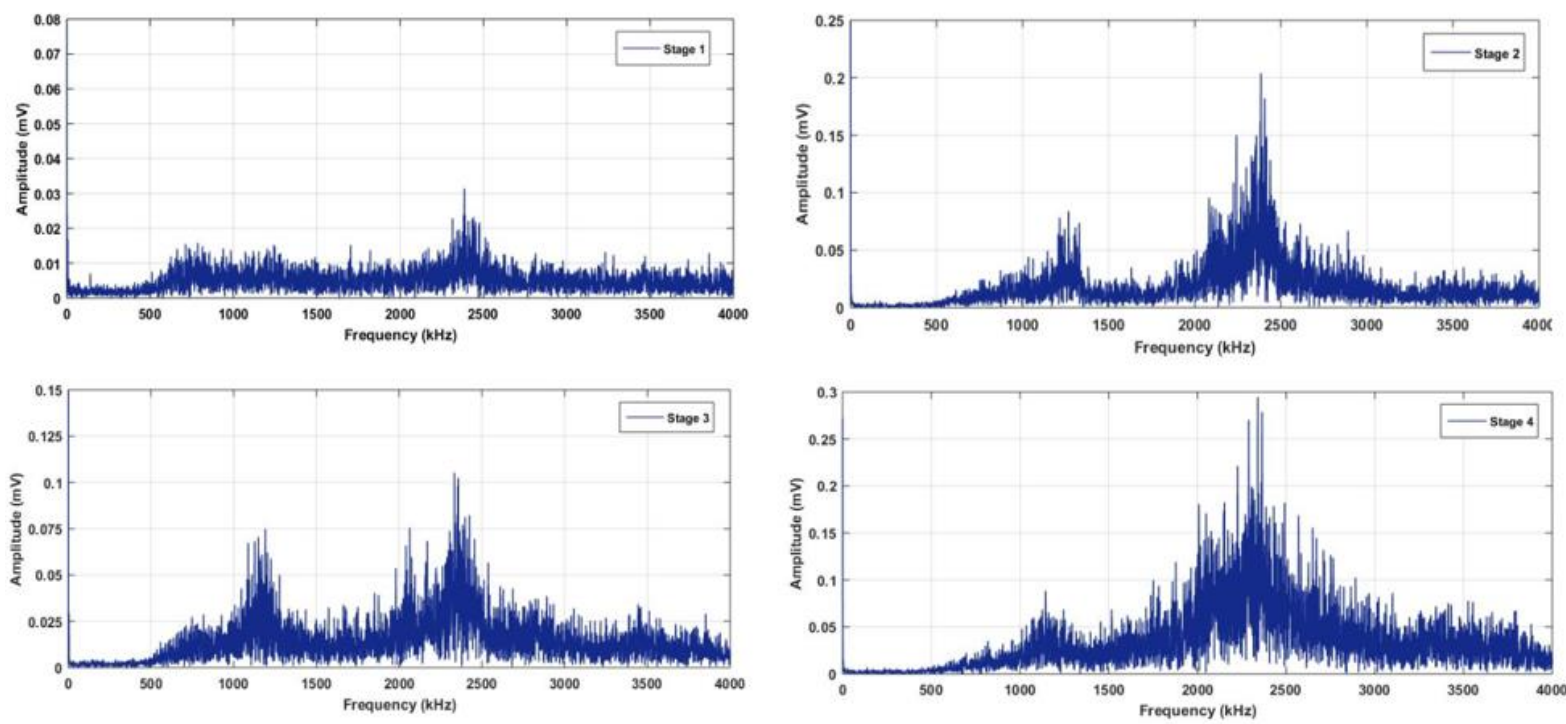

Figure 8: Frequency plot of $A E$ amplitude for sensor 1 for tap water, water level of $200 \mathrm{~mm}$.

The AE signal grew substantially during stage 4, reaching its highest level of $0.3 \mathrm{mV}$ at $2300 \mathrm{kHz}$. The smaller peak at $1250 \mathrm{kHz}$ also increased in magnitude. During this stage, there was a lot more bubble activity. Additionally, this stage presents liquid entrainment, a phenomenon that occurs with nucleate boiling when the bubbles rise to the water's free surface and carry with them hot water which helps increase the overall water temperature. The bubbles to grow and rise to the water's free surface under the influence of buoyancy. Then the bubbles burst and release the contained vapour. As a result, the heat flux reaches its maximum value. Observations from the spectra of the AE signal note the presence of more bubble formation at a faster rate, as shown in Figure 8, stage 4. Typically, AE signals associated with bubble activity increased throughout the boiling test.

\subsection{Observation of the influence of different liquid types on AE signal levels}

Figure 9 below shows the AE-RMS $(\mathrm{mV})$, time (sec) and temperature $\left({ }^{0} \mathrm{C}\right)$ for sensor 1 , where the black line represents tap water, the red line demineralized water, and the green line salt water $(5 \mathrm{~g} / \mathrm{L})$. The plots show clear differences between the three 
liquids. With tap water, bubbles start to detach from the heated surface of the boiler at around $245 \mathrm{~s}$, and $30^{\circ} \mathrm{C}$. In addition, for tap water, gas voids appear on the boundaries between the water and the small particles suspended in the water; an event called heterogeneous nucleation.

For demineralized water, the bubbles start to detach from the heated surface at about $570 \mathrm{~s}$, at a water temperature of about $50^{\circ} \mathrm{C}$. In demineralized water there are far fewer small particles in the liquid on which heterogeneous nucleation can occur. However, the thermal motion inside the demineralized water formed temporary, microscopic voids which by themselves can produce the nuclear cavitation necessary for formation and growth of micro-bubbles. An increase in water temperature meant the local saturation vapour pressure became greater than the surrounding water pressure and bubbles formed.

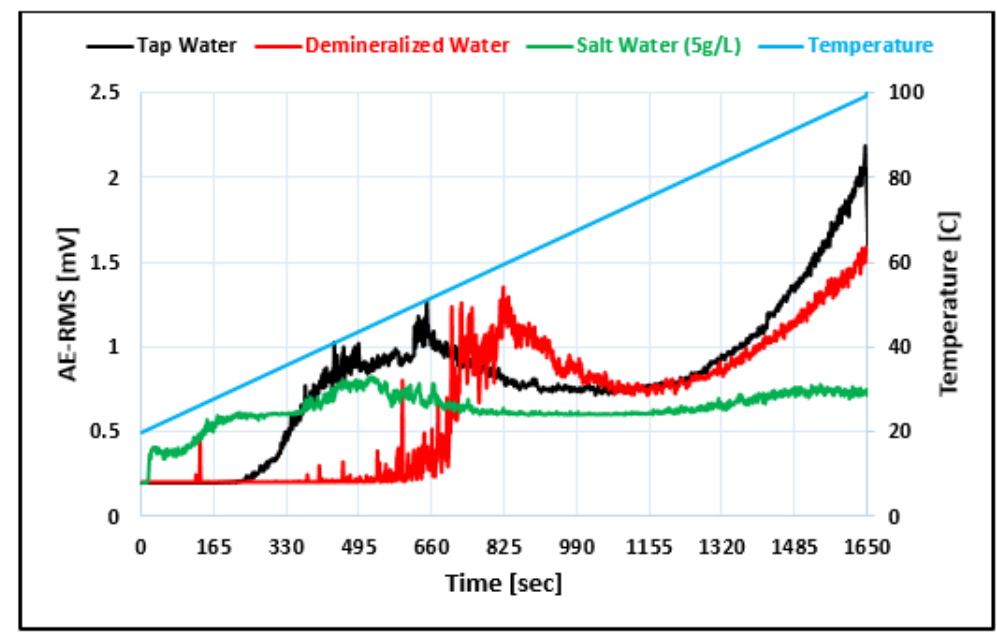

Figure 9: Measured temperature, and AR-RMS signals from sensor 1 for boiling test with tap water, demineralized water and salt water $(5 \mathrm{~g} / \mathrm{L})$ (water level $200 \mathrm{~mm}$ ).

From Figure 9 it can be seen that bubbles detach from heated surface to the water surface in the salt water faster than tap water or demineralized water. With salt water $(5 \mathrm{~g} / \mathrm{L})$, bubbles start to detach from the heated surface into the surrounding water at a very early stage, from about $22^{\circ} \mathrm{C}$. It was observed that detachment of bubbles from the heated surface at these relatively low temperatures took place in salt water $(5 \mathrm{~g} / \mathrm{L})$ but not in either tap water or demineralized water. This is due to the surface tension of salt water $(5 \mathrm{~g} / \mathrm{L})$ being higher than that of the other two liquids. Additionally, bubble voids appear on the boundary between the liquid and small salt particles suspended in the liquid, which is responsible for formation and growth of micro-bubbles, providing what is called a flat hydrophilic surface [29]. 
The mechanism of bubble formation in tap water is the same as in salt water, but there is a difference in the size of the bubbles. In tap water, many large bubbles appeared and disappeared in a short time. However, no large individual bubbles were observed in the salt water.

It can be seen that the detachment of bubbles from the heated surface with tap water is faster than for demineralized water because in demineralized water, there is a much lower concentration of mineral ions and other small particles [29][40].

\subsection{Statistical analysis of AE parameters of bubble formation during pool boiling with different liquid types}

Bubble formation during pool boiling depends liquid properties such as viscosity, density and surface density. The AE signals corresponding to bubble formation were statistically analysed to determine a critical AE parameter indicator for bubble activity detection. Table 2 shows average values for comparison between AE Amplitude, AE Rise time, AE Count and AE Energy obtained from sensor 1 (for bubble formation, over time interval was $1650 \mathrm{~s}$ ). These statistical parameters were obtained directly from the AE system (AEWin).

Table 2: Comparison of average AE parameter of bubble formation with different liquid types during water level of $200 \mathrm{~mm}$ at time limited which is $1650 \mathrm{~s}$.

\begin{tabular}{|l|c|c|c|c|}
\hline \multirow{2}{*}{ Liquid Types } & \multicolumn{4}{|c|}{ AE Parameters } \\
\cline { 2 - 5 } & $\begin{array}{c}\text { AE Amplitude } \\
(\mathrm{dB})\end{array}$ & AE Rise Time ( $\mu \mathrm{s})$ & AE Count & AE Energy (atto-joule) \\
\hline Tap Water & 38 & 701 & 29 & 5 \\
\hline Demineralized Water & 38 & 1133 & 75 & 8 \\
\hline Salt Water (5g/L) & 37 & 27 & 4 & 0.33 \\
\hline
\end{tabular}

The AE signals presented in Table 2 were the average of 15 tests based on the raw data from the AE system. One recommendation could be the use of waveform analysis for the transient signal instead of taking data from the whole waveform (statistical AE parameters from the AE system). A comparison of bubble formation in tap water, demineralized water and salt water $(5 \mathrm{~g} / \mathrm{L})$, is shown in Figures 10,11 , and 12. The results are for $A E$ Rise time, $A E$ Count and $A E$ Energy. 


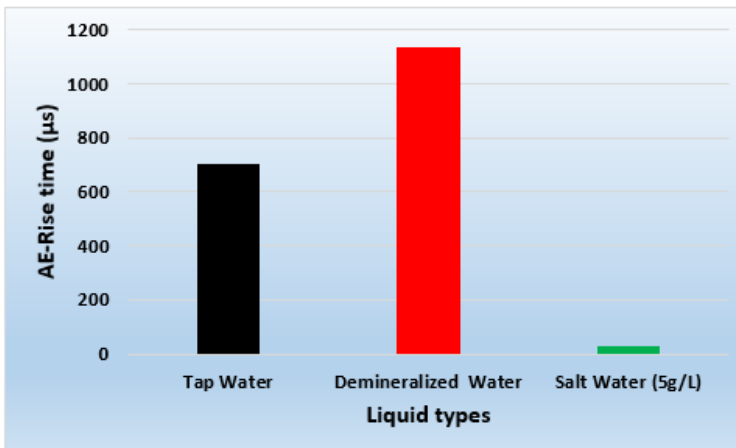

Figure 10: AE Rise time from bubble formation as a function of liquid types.

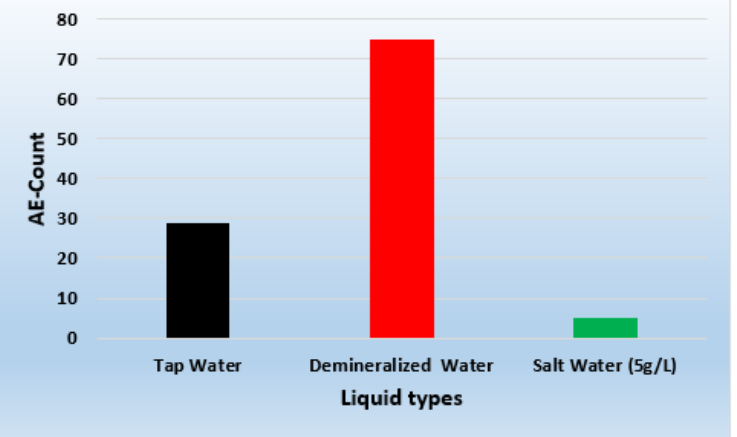

Figure 11: AE Count from bubble formation as a function of liquid types.

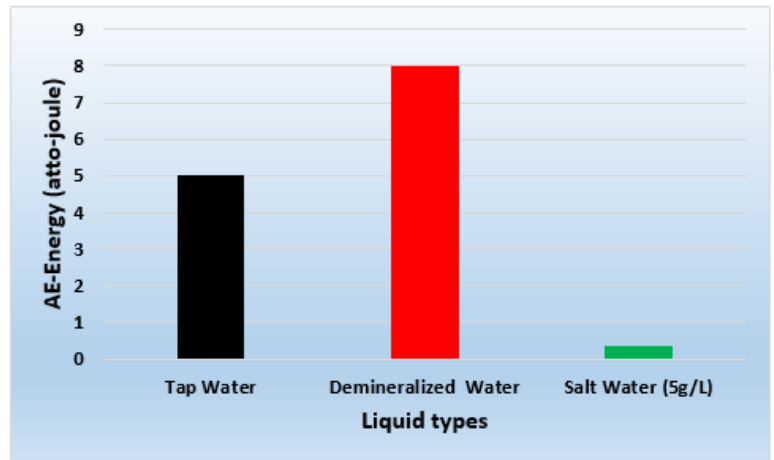

Figure 12: AE Energy from bubble formation as a function of liquid types.

Figures 10,11, and 12, show a significant effect of viscosity on bubble activity and the AE Energy generated. (Where are viscosity values?) The AE signal level increased as viscosity decreased. These figures show that for demineralised water the count, energy level and rise time were consistently much the greatest, and for salt water were much the lowest. This is because the bubbles size in salt water bigger than that of demineralised water.

Figure 13 shows the $A E$ amplitude decreases with different liquid types. However, the value of $A E$ amplitude for tap water is $38 \mathrm{~dB}$, the same as for demineralized water. These the levels correspond to stage 2 in Figure 9 


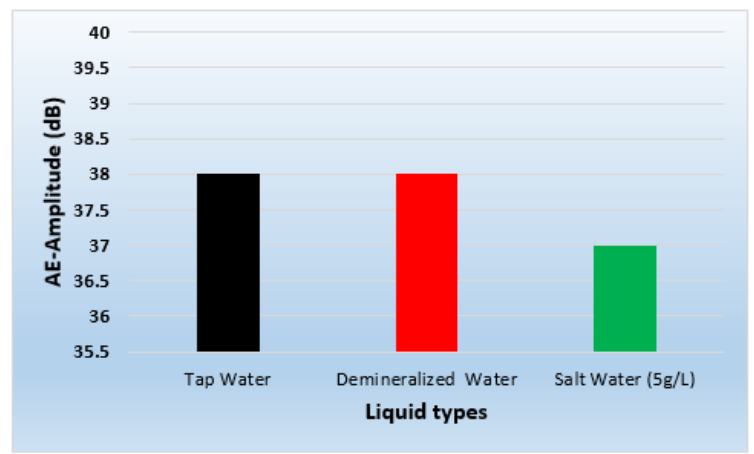

Figure 13: AE Amplitude from bubble formation as a function of liquid.

\subsection{Observation of the influence of the level of the tap water on AE signals}

Figure 14 shows the relationship between the AE-Energy (atto-joule) measured by sensor 2 with water temperature $\left({ }^{0} \mathrm{C}\right)$ measured by the thermocouple, for a (tap) water surface level of $100 \mathrm{~mm}$, some $200 \mathrm{~mm}$ below sensor 2. The AE-Energy was only 200 atto-joules for a water temperature of $100^{\circ} \mathrm{C}$, this was because sensor 2 was $200 \mathrm{~mm}$ above the water surface and the AE signal was transmitted to the sensor only via the wall of the container.
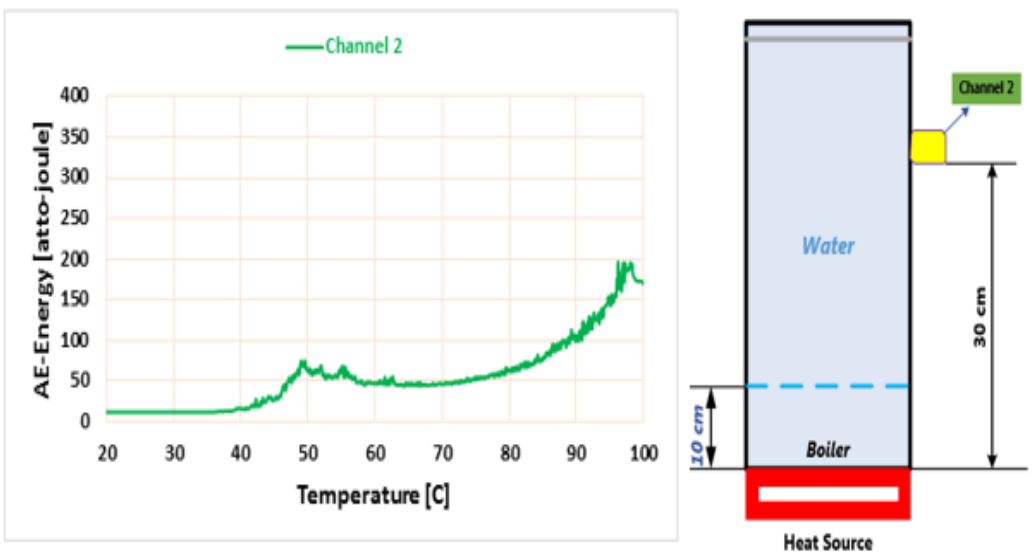

Figure 14: AE-Energy measured at sensor 2 with (tap) water level of $100 \mathrm{~mm}$ from the bottom of the boiler.

Figure 15, shows the same process but with the (tap) water surface level at $200 \mathrm{~mm}$, only $100 \mathrm{~mm}$ below the sensor. The value of $A E$-Energy signal increased to approximately 250 atto-joules for a water temperature of $100^{\circ} \mathrm{C}$, this small increase was due to the water level being closer to the sensor and the AE signal not having to travel so far through the wall of the container. 


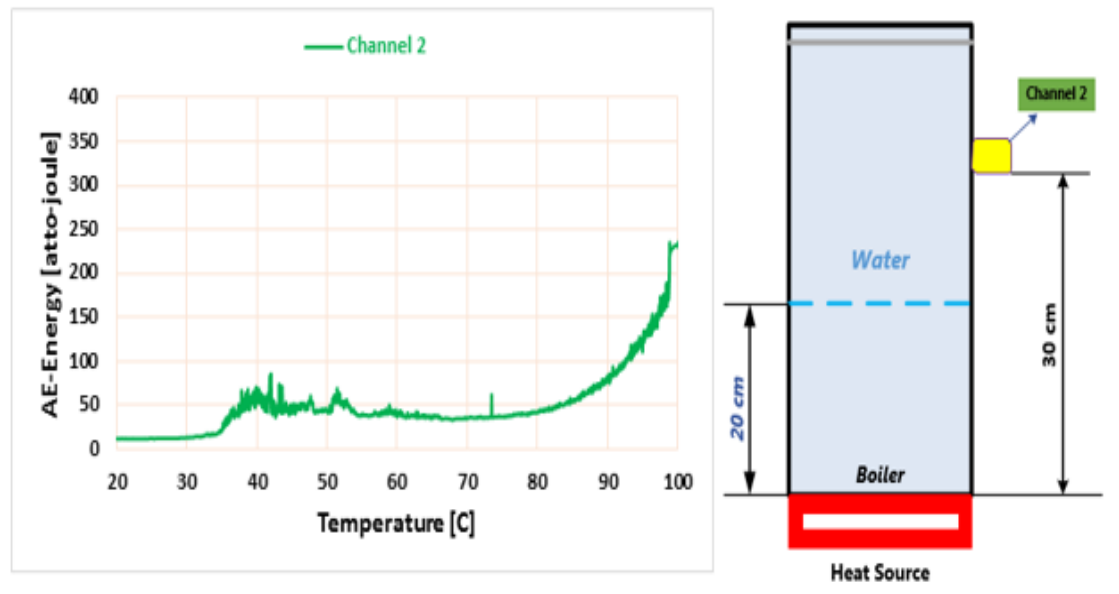

Figure 15: AE-Energy measured at sensor 2 with (tap) water level of $200 \mathrm{~mm}$ from the bottom of the boiler.

Figure 16 again shows the same process but now the water depth is $350 \mathrm{~mm}$ which is $50 \mathrm{~mm}$ above the sensor position. There is a substantial increase in the measured AE-Energy, about 400 atto-joules when the water approached the boiling phase.

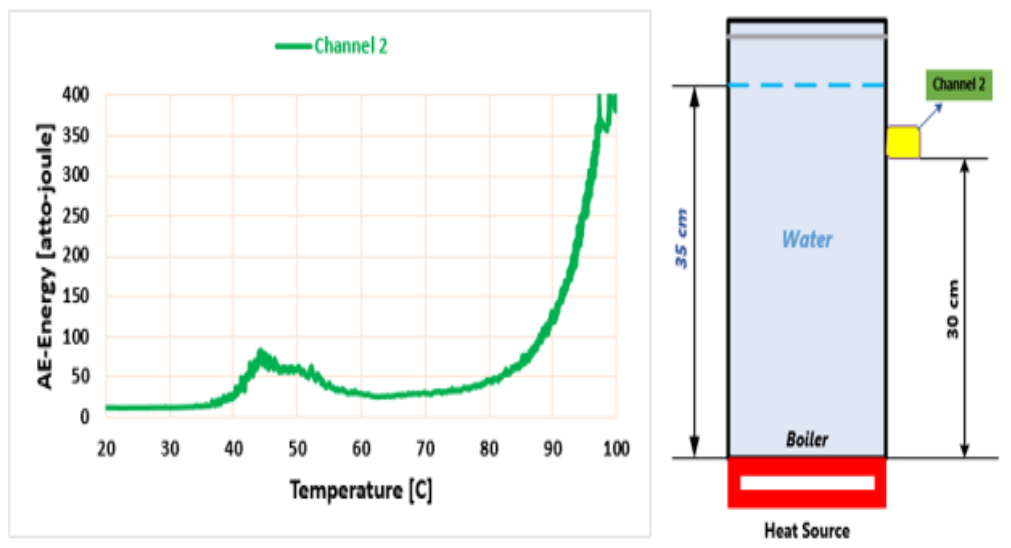

Figure 16: AE-Energy measured at sensor 2 with (tap) water level of $350 \mathrm{~mm}$ from the bottom of the boiler.

Obviously, the water level has a substantial effect on the measured level of $A E$ energy with pool boiling. When the water level is below the level of the sensor direct transmission from water to sensor is not present and the measured signal is only that transmitted through the wall of the container. Thus, as expected, there is a marked drop in amplitude of the signal when the water level falls below that of the sensor.

The maximum value of $A E$-Energy is recorded between 90 and $100^{\circ} \mathrm{C}$ for all levels because more bubbles form at these temperatures and their energy increases. 


\subsection{Threshold levels of AE signals using tap water}

Figure 17 below shows that there is a correlation between a number of hits and level of the water in the container. When the liquid level rises, the number of hits also increases due to the increased number of bubbles formed. (Are you sure the rate at which bubbles were produced increased with water level. I would have thought the opposite was the case because the pressure on the heated surface gets greater with increased water level and so bubbles won't form until a slightly higher temperature. Surely, what is happening is that when the water level is below sensor 2 the signals are attenuated, don't reach the $36 \mathrm{~dB}$ threshold level and are not counted.)

The value of AE-Hits reached 160,000 hits for a water depth of $350 \mathrm{~mm}$, and threshold of $36 \mathrm{~dB}$. As would be expected, the number of hits decreased with increase in threshold level, as shown in Figure 17.

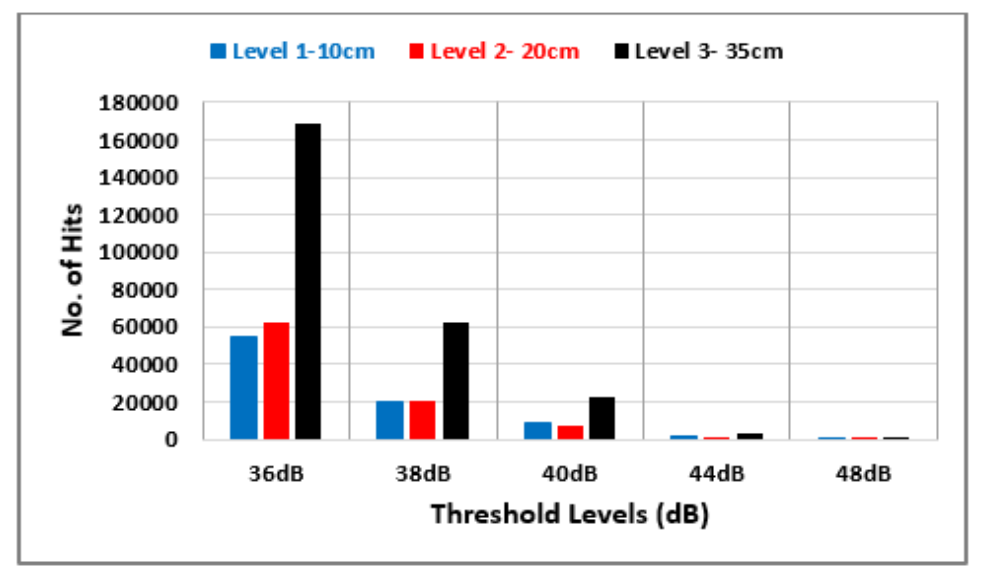

Figure 17: Effect of level of (tap) water and threshold levels on number of AE-hits for channel 2

\subsection{Energy index of AE signals using tap water}

The next phase of the analysis was the use of the Energy Index (EI) to identify bubble formation during the test period with different rates of power-supply to the plate heater. The Energy Index (EI) is defined as the square of the ratio of root mean square of a defined segment ( $\left(\mathrm{RMS}_{\text {segment }}\right)$ of a given signal to the root mean square

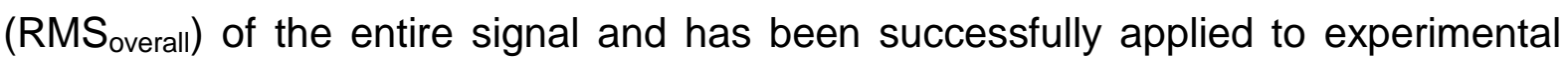
data from gears, bearings, and bubble activity [8][41][42]. For this particular research investigation every AE waveform recorded throughout the tests, was divided into 1650 segments and El was calculated using Equation (5). 


$$
\text { Energy Index }=\left(\frac{R M S_{\text {segment }}}{R M S_{\text {overall }}}\right)^{2}
$$

Figure 18 shows the correlation between energy index and measured water temperature $\left({ }^{0} \mathrm{C}\right)$. The blue line represents the $A E$ signal for a $3.0 \mathrm{~kW}$ supply to the heater and the black line shows the AE signal for the $2.5 \mathrm{~kW}$ supply.

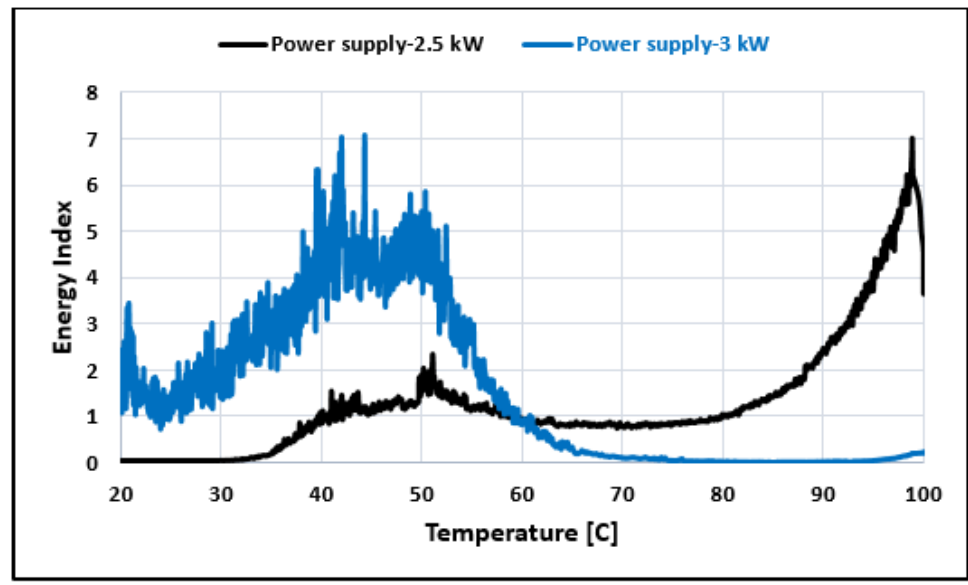

Figure 18: Effect of power-supply on the AE levels with (tap) water depth of $200 \mathrm{~mm}$ for sensor 1 .

From Figure 18, it can be seen that for a heater of $2.5 \mathrm{~kW}$ power, it is obvious that the El plot follows very closely the AE-RMS plot presented in Figure 5, for sensor 1. There is an increase in El level with measured water temperatures and bubbles started to detach from the heated surface to the surrounding water at about $35^{\circ} \mathrm{C}$. The $\mathrm{El}$ of the $\mathrm{AE}$ signal decreased slowly between 50 and $80^{\circ} \mathrm{C}$, following the plot shown in Figure 5. In this period, attenuation occurs due to some bubbles sticking to the heated surface of the boiler, causing of reduction in the $A E$ signal. In the last moments of the test $\left(100^{\circ} \mathrm{C}\right)$, large bubbles were bursting at the free surface, and the corresponding value of the $\mathrm{El}$ at $2.5 \mathrm{~kW}$ was recorded as 7 , (see Figure 18).

With a power supply of $3 \mathrm{~kW}$, the bubbles start to detach from the heated surface at the bottom of the boiler at around $22^{\circ} \mathrm{C}$ with a substantial increase in the level of the $\mathrm{El}$ between 22 and $50^{\circ} \mathrm{C}$. The $\mathrm{El}$ level is much greater than for the $2.5 \mathrm{~kW}$ source, with increased power supply generating a higher heat flux and causing more bubbles to form. The level of value of the $\mathrm{El}$ for the $3 \mathrm{~kW}$ heater peaked at 7 , and this occurred between 40 and $50^{\circ} \mathrm{C}$, because in this period the bubbles formed at a faster rate, due to the increased heat flux and heated surface temperature. After that, there was a gradual decrease in the value of El with increase in temperature from 50 
to $100^{\circ} \mathrm{C}$. During this period, the water temperature and bubble size increased, causing bubbles to burst near the free surface.

It was observed that the power-supply has a significant effect on AE levels such that when the power supply increases, the trend of the AE signal changes as well.

\subsection{Difference between rise times of AE-signals for bubble formation and bubble burst using tap water}

Figure 19 shows a schematic of the measurement positions for determining the difference between $\mathrm{AE}$-signals associated with bubble formation and bubble burst. To measure the signals associated with bubble formation, acoustic waveguide 1 with sensor attached was positioned with its open end $5 \mathrm{~mm}$ from the bottom (heated) surface of the boiler. To monitor bubble burst at the surface, acoustic waveguide 2 was placed with its open end $195 \mathrm{~mm}$ from the bottom heating surface. The AE signal rise times for waveguide 1 were greater than those for waveguide 2; $6494 \mu \mathrm{s}$ compared to $1753 \mu \mathrm{s}$. This increase in rise time is because as the bubbles neared the surface their diameter increased, the bubbles became larger. The biggest bubbles burst on the surface of the water at the end of the test.
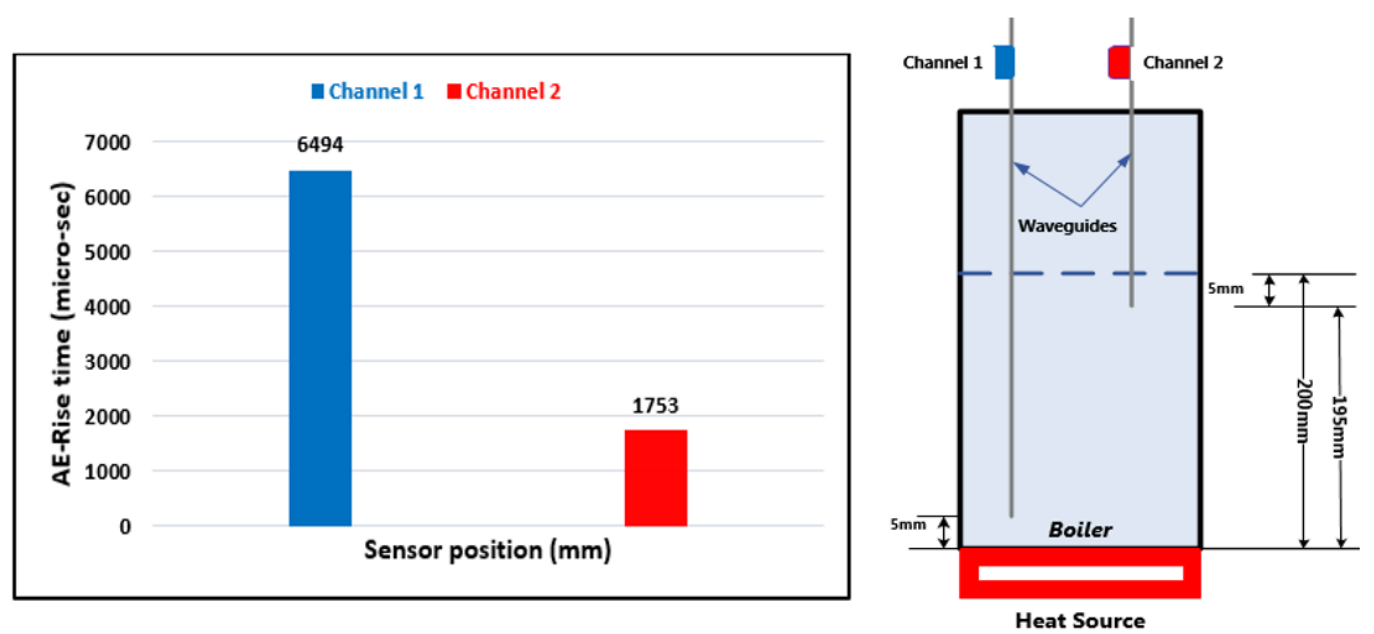

Figure 19: AE-rise times for different waveguide positions using tap water at $3 \mathrm{~kW}$ supply.

The identical set up was used to measure AE-RMS to identify characteristics of bubble activity, such as bubble formation and burst, as shown in Figure 20. This analysis was performed to assess the ability of the RMS parameter to monitor bubble formation and burst during pool boiling. The results showed the AE signal for 
waveguide 1 was higher than that for waveguide $2 ; 3.5 \mathrm{mV}$ and $0.7 \mathrm{mV}$ respectively at $40^{\circ} \mathrm{C}$, see Figure 20 . The peak values for waveguide 1 , measuring activity $5 \mathrm{~mm}$ above the heated surface, was for the temperature range 30 to $50^{\circ} \mathrm{C}$, in the time period 300 to $700 \mathrm{~s}$ (see Figure 5). In this period, there was greater bubble formation on the heated surface with a few bubbles bursting on the water's free surface as bubbles started to detach from the heating surface of the boiler vessel into the surrounding water.
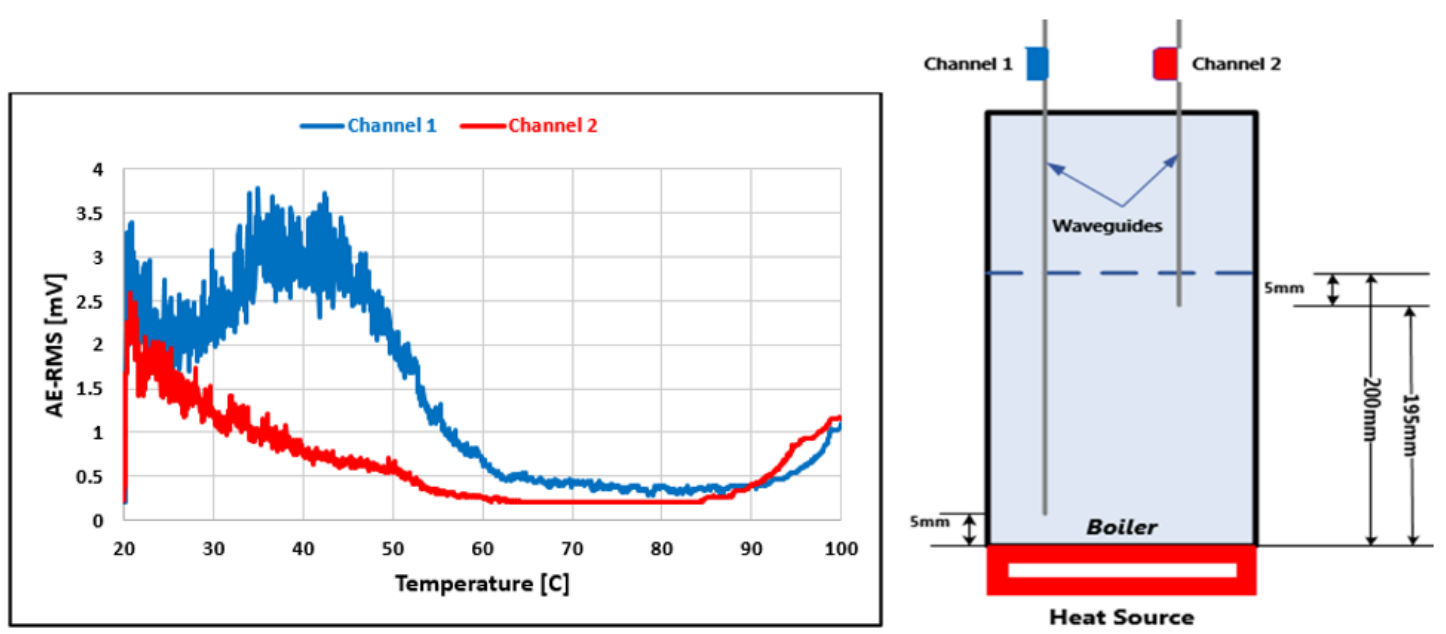

Figure 20: AE-RMS for different waveguide positions using tap water at $3 \mathrm{~kW}$ supply.

It was noted that the $A E$ signal levels for waveguide 1 gradually decreased to approximately $0.4 \mathrm{mV}$ between $55^{\circ} \mathrm{C}$ and $80^{\circ} \mathrm{C}$ but picking up slightly when the temperature rose above $95^{\circ} \mathrm{C}$. During this stage, some of the bubbles combined with adjacent bubbles and stuck to the heated surface of the boiler, causing attenuation of $A E$ signals.

As the water approached the boiling phase between 95 and $100^{\circ} \mathrm{C}$, a gradual increase in $\mathrm{AE}$ signal levels to around $1 \mathrm{mV}$ for both waveguides was observed, see Figure 20. This increase was attributed the heat gained by the water. Furthermore, this heat caused a significant departure of the bubbles towards the top surface, and finally, large bubbles began to burst on surface as both their size and internal energy increased. These results lead to the hypothesis that the AE-signals of bubble formation are higher and more violent than those of bubble burst at the free surface.

\section{Conclusions}


$\mathrm{AE}$ techniques are capable of detecting the formation and bursting of bubbles in the early stages of the boiling process. Furthermore, this study has demonstrated that AE parameters such as AE-RMS, AE-Energy, AE-Amplitude, AE Energy Index, and AE Rise Time are reliable, robust and sensitive for the detection of bubble occurrence and propagation to the surface of the water during pool boiling. It was concluded that the condition monitoring of bubble formation using $A E$ techniques could usefully complement other condition monitoring technologies, all of which are aimed at reducing energy losses and improving life-cycle costs.

This paper presents the results of an investigation of the application of $\mathrm{AE}$ technology to the monitoring of early stage bubble formation in pool boiling. This project has established that different liquids will affect the $A E$ energy levels during bubble formation. It was noted that there is a correlation between $A E$ signals and water levels; when the water level increases, the value of $A E$ signal increases.

Obtained results are shown that the AE signal can be usefully used for the diagnosis and monitoring of bubble generation rate in the early stages of pool boiling and detection of the level of the fluid contained in a heated vessel. Furthermore, the $A E$ signal can differentiate between similar liquid - which means that the AE signal might be used to detect the deterioration in boiling process.

\section{References}

1. Jazi a. M., Rahimzadeh $\mathrm{H}$. Waveform analysis of cavitation in a globe valve. Ultrasonics. Elsevier B.V.; 2009; 49(6-7): 577-582. Available at: DOI:10.1016/j.ultras.2009.02.004

2. Brennen C. Hydrodynamics of Pumps. Oxford Uni. Cambridge University Press; 1994. Available at: DOI:10.1017/CBO9780511976728

3. Jazi A. Masjedian, and Rahimzadeh $\mathrm{H}$. Detecting cavitation in globe valves by two methods: Characteristic diagrams and acoustic analysis. Applied Acoustics. Elsevier Ltd; 2009; 70(11-12): 1440-1445. Available at: DOI:10.1016/j.apacoust.2009.04.010

4. Neill, G.D., Reuben, R.L. and Sandford PM. Detection of Incipient Cavitation in Pumps using Acoustic Emission. Journal of Process Mechanical Engineering. 1979; 211(4): 267-277.

5. Husin S., Addali A., Mba D. Feasibility study on the use of the Acoustic Emission technology for monitoring flow patterns in two phase flow. Flow Measurement and Instrumentation. Elsevier; 2013; 33: 251-256. Available at: DOI:10.1016/j.flowmeasinst.2013.07.011

6. Alfayez L., Mba D., Dyson G. The application of acoustic emission for 
detecting incipient cavitation and the best efficiency point of a $60 \mathrm{~kW}$ centrifugal pump: Case study. NDT and E International. 2005; 38(5): 354-358. Available at: DOI:10.1016/j.ndteint.2004.10.002

7. Carmi, R., Bussiba, A., Widenfeld, G., Aharon, Y., Alon I., and Hochbaum, I. Detection of Transient Zones During Water Boiling by Acoustic Emission. Acoustic Emission. 2011; 29: 89-97.

8. Alhashan T., Elforjani M., Addali A., Teixeira J. Monitoring of Bubble Formation during the Boiling Process Using Acoustic Emission Signals. International Journal of Engineering Research \& Science (IJOER). 2016; 2(4): 66-72.

9. Benes P., Uher M. Identification of liquid boiling by acoustic emission. Fundamental and Applied Metrology. 2009; (1): 1396-1401.

10. Baek SH., Wu K., Shim HS., Lee DH., Kim JG., Hur DH. Acoustic emission monitoring of water boiling on fuel cladding surface at 1 bar and 130 bar. Measurement: Journal of the International Measurement Confederation. Elsevier Ltd; 2017; 109: 18-26. Available at: DOI:10.1016/j.measurement.2017.05.042

11. Alhashan T., Addali A. The Effect of Salt Water on Bubble Formation during Pool Boiling Using Acoustic Emission Technique. 2016; 13(5): 51-56. Available at: DOI:10.9790/1684-1305085156

12. Jaubert L., Maillard S., Sulis V. Monitoring of Fluidic Systems By Acoustic Emission. European Conf. AE Testing. France; 2008. pp. 334-339.

13. Alfayez, L., D. Mba and G dyson. Detection of incipient cavitation and the best efficiency point of 2.2MW centrifugal pump using acoustic emission. in 26th Eu. Berlin; 2004.

14. Osterman A., Hočevar M., Širok B., and Dular M. Characterization of incipient cavitation in axial valve by hydrophone and visualization. Experimental Thermal and Fluid Science. 2009; 33(4): 620-629. Available at: DOI:10.1016/j.expthermflusci.2008.12.008

15. Shuib H., An experimental investigation into the correlation between Acoustic Emission (AE) and bubble dynamics. Cranfield University; 2011.

16. Alssayh MA. Slug Velocity Measurement and Flow Regime Recognition Using Acoustic Emission Technology. PHD Thesis. Cranfield University; 2013.

17. FDIS ISO. International Standards Organization Documents 22096. Documents. ISO, Generic RT (eds.) Condition monitoring and diagnosis of machines - Acoustic Emission; 2007.

18. Corporation PA. AE win PCl-2 Based AE System User's Manual. Mistras Group Inc. 2007; REV 3(April): 1-312.

19. Alssayh M., Addali A., Mba D., El-Alej ME. Slug velocity measurement using acoustic emission technology. Proceedings of the Institution of Mechanical Engineers, Part E: Journal of Process Mechanical Engineering. 2014; 230(1): 76-83. Available at: DOI:10.1177/0954408914532244

20. Elasha F., Greaves M., Mba D., Fang D. A comparative study of the effectiveness of vibration and acoustic emission in diagnosing a defective bearing in a planetry gearbox. Applied Acoustics. Elsevier Ltd; 2017; 115: 181-195. Available at: DOI:10.1016/j.apacoust.2016.07.026 
21. Fowler TJ. Chemical industry applications of acoustic emission. Materials Evaluation. 1992; : 875-882.

22. Alhashan T., Addali A., and Amaral J. Experimental investigation of the influences of different liquid types on acoustic emission energy levels during the bubble formation process. International Journal of Energy and Environmental Engineering. Springer Berlin Heidelberg; 2017; Available at: DOI:10.1007/s40095-017-0245-5

23. Addali A. Monitoring gas void fraction in two-phase flow with Acoustic Emission. PhD Thesis. Cranfield University; 2010.

24. Marto, P. J., and Rohsenow WM. Effects of Surface Conditions on Nucleate Pool Boiling of Sodium. ASME J. Heat Transfer. 1966; 88: 196-204.

25. Minnaert, M. On Musical Air-Bubbles and the Sounds of Running Water. Philosophical Mag. 1933; 16: 235-248.

26. Kloeppel, JE. Scientists measure energy dissipation in a single cavitating bubble. Physical Sciences. 2002; 217: 244-1073.

27. Leighton, TG. The acoustic bubble: Oceanic bubble acoustics and ultrasonic cleaning. Proceedings of Meetings on Acoustics. 2015; 24: 1-5. Available at: DOI:10.1121/2.0000121

28. Derakhshan, O, Houghton, J., Jones, R., and March, P. Cavitation monitoring of hydro turbines with RMS acoustic emission measurements. Proceedings of the world meeting on acoustic emission. 1989; 15: p.305.

29. Christopher, Brennen E. Cavitation and bubble dynamics. Oxford: Oxford University Press; $1995.145-185$ p. Available at:

DOI:10.1017/CBO9781107338760

30. Sinha. N. Characterization of Liquids Using Gas Bubbles. United States Patent No: US 7,010,962 B2. 2006;

31. Cengel Y a. Heat Transfer: A Practical Approach. Mc Graw-Hill. 2003. 785-841 p.

32. Blanchard, D. C., and Woodcock, A. Bubble Formation in Modification in the Sea and its Meteorological Signifcance. Tellus. 1957; vol.9, NO.: 145-158.

33. Abe, T. On the Stable Formation of Sea Water in Seas. journal of the oceanographical society of Japan. 1962; 20: 242-250.

34. Ceccio, S., Gowing, S., and Shen, Y. The Effects of Salt Water on Bubble Cavitation. Journal of Fluids Engineering. 1997; Vol. 119: 155-163.

35. Steven, F. Encyclopedia of Mathematics and its Applications. Cambridge-UK: The Press Syndicate of the University of Cambridge; 2003.

36. Lihui, W GR. Condition Monitoringand Control for Intelligent Manufacturing. USA: University of Michigan; 2006.

37. Boczar T., and Lorenc, M. Determinig the repeatability of acoustic emission generated by the Hsu-Nielsen calibrating source. Molecular and Quantum Acoustics. 2004; 25: 177-192.

38. Elforjani, M. Condition Monitoring of Slow Speed Rotating Machinery Using Acoustic Emission Technology. Cranfield Univesity; 2010. Available at: DOI:10.1017/CBO9781107415324.004 
39. Husin, S., Addali a., Mba, D. Observation of acoustic emission from gas bubble inception and burst. Proceedings of the Institution of Mechanical Engineers, Part E: Journal of Process Mechanical Engineering. 2012; 226(1): 79-88. Available at: DOI:10.1177/0954408911409518

40. Mitta, KL. Advances in Contact Angle, Wettability and Adhesion. Canada: Scrivener Publishing LLC. All rights reserved; 1983.

41. Al-Balushi, KR., Addali A., Charnley B., Mba, D. Energy index technique for detection of acoustic emissions associated with incipient bearing failures. Applied Acoustics. Elsevier Ltd; 2010; 71(9): 812-821. Available at: DOI:10.1016/j.apacoust.2010.04.006

42. Elforjani M., Mba, D. Observations and location of acoustic emissions for a naturally degrading rolling element thrust bearing. Journal of Failure Analysis and Prevention. 2008; 8(4): 370-385. Available at: DOI:10.1007/s11668-0089141-x 